\title{
Recent data on zebra patterns
}

\author{
G. P. Chernov ${ }^{1,2, \star}$, Y. H. Yan ${ }^{1}$, Q. J. Fu ${ }^{1}$, and Ch. M. Tan ${ }^{1}$ \\ 1 National Astronomical Observatory, Chinese Academy of Science, 20A, Datun Road, Beijing, 100012, PR China \\ e-mail: yyh@bao.ac.cn \\ ${ }^{2}$ Izmiran, Troitsk, Moscow region, 142190, Russia \\ e-mail: gchernov@izmiran.rssi.ru
}

Received 20 December 2004 / Accepted 13 February 2005

\begin{abstract}
A comparative analysis of two recent solar radio outbursts around $3 \mathrm{GHz}$ with zebra structures and fiber bursts in their dynamical radio spectra is carried out using all available ground-based and satellite data (SOHO, TRACE, RHESSI). The latest theoretical models of the zebra pattern are critically discussed. New data on microwave zebra structures and fiber bursts suggests that they are analogous to similar structures observed at meter wavelengths. It was discovered that in the $2,6-3,8 \mathrm{GHz}$ frequency band more than 34 zebra stripes can appear simultaneously, and some isolated fiber bursts can continuously be transformed into zebra stripes. This fact indicates a single origin for both structures. The zebra pattern was observed when the signs of magnetic reconnections were revealed in images of $195 \AA$ AV lines, and radio sources coincided with positions of some new sources in hard X-rays. All the main properties of the stripes in emission and absorption can be explained if they are associated with interactions between electrostatic plasma waves and whistlers, taking into account the quasi-linear diffusion of fast particles with the loss-cone distribution on whistlers. In this model it is possible to obtain realistic values for the magnetic field strength of $B \approx 160 \mathrm{G}$ at the plasma level of about $3 \mathrm{GHz}$. The double plasma resonance model for the zebra pattern based on the known realistic dependences of electron density and magnetic field yields a frequency dependence for the frequency separation between stripes that does not agree with the observations.
\end{abstract}

Key words. Sun: flares - Sun: radio radiation - Sun: activity - Sun: UV radiation

\section{Introduction}

Stripes in emission and absorption called zebra pattern structure (ZS) and fiber-bursts (FB) (or bursts with intermediate frequency drift) are well-known fine structures seen in the meter and decimeter continuum emission of type IV solar radio bursts (Elgarøy 1959; Slottje 1972; Kuijpers 1975; Slottje 1981; Elgarøy 1982). High time and frequency resolution data have improved our studies of similar fine structures in star flares. Some recent observations with a time resolution of $0.1 \mathrm{~s}$, using Ondřejov radio spectrographs in the $0.8-4 \mathrm{GHz}$ range, have allowed us to find new properties of zebra structures and to verify theoretical models (Ledenev et al. 2001; Karlicky et al. 2001; Sawant et al. 2002). The new microwave spectrometers of NAOC (Huairou station, China) with higher resolution (10 MHz and 5-8 ms) (Fu et al. 1995) can observe detailed zebra patterns and fiber bursts at high frequencies, $2.6-7.6 \mathrm{GHz}$ (Chernov et al. 2001, 2003). The same variety of fine structure is observed in the microwave range.

In the course of development of the interpretation of such fine structures, the theory always lagged behind the observational data obtained (Kuijpers 1980). The interaction of plasma

^ Constant address: IZMIRAN, Troitsk, Moscow region, 142190, Russia. electrostatic waves $(l)$ with whistler waves $(w)$ (generated by the same fast particles with loss-cone anisotropy) is a wellaccepted emission mechanism for fiber bursts: $l+w \rightarrow t$ with freely escaping electromagnetic waves $(t)$ in the ordinary $(\mathrm{O})$ mode (Kuijpers 1975). A more detailed analysis of the interaction $l+w \rightarrow t$ was made in Fomichev \& Fainstein (1988), where it was shown that the observed fiber radio fluxes are explained in the framework of weak plasma turbulence.

Zebra patterns are more complicated structures, and many mechanisms have been proposed to explain them (Kuijpers 1975; Zheleznykov \& Zlotnik 1975a,b; Chernov 1976; Mollwo 1983, 1988; Winglee \& Dulk 1986). The majority are based on electrostatic emission at double plasma resonance (DPR):

$\omega_{\mathrm{UH}}=\left(\omega_{\mathrm{Pe}}^{2}+\omega_{\mathrm{Be}}^{2}\right)^{1 / 2}=s \omega_{\mathrm{Be}}$

with $\omega_{\mathrm{UH}^{-}}$upper hybrid frequency, $\omega_{\mathrm{Pe}^{-}}$electron plasma frequency, $\omega_{\mathrm{Be}}$ - electron cyclotron frequency and s- integer harmonic number. The most advanced model in this category, proposed by Wenglee \& Dulk (1986), is based on cyclotron non-saturated maser emission of electrostatic waves by a losscone electron distribution. They assume that the theory of Zheleznyakov \& Zlotnik (1975a) is restricted due to the use of the narrow distribution function that peaks at high pitch angles. 
However, some difficulties remain with all these versions: the frequency separation between zebra stripes $\Delta f_{\mathrm{e}}$ must comprise a certain portion of the electron cyclotron frequency (depending on the relationships of the height scales of density and magnetic field in the corona), and this is hard to reconcile with its often irregular variation with frequency; the magnetic field deduced from $\Delta f_{\mathrm{e}}$ seems too low for the flare region and we are faced with the difficulty of having plasma $\beta \approx\left(v_{\mathrm{s}} / v_{\mathrm{A}}\right)^{2} \leq 1$ (a well-accepted value for magnetic traps in active regions). Practically all models explain only stripes in emission, although the stripes are sometimes observed predominantly in absorption. An important property of the losscone distribution was missed in all models: it also generates whistlers, and interaction of whistlers with fast particles radically changes the velocity distribution function when the transverse anisotropy decreases, but a beam on the longitudinal velocities appears (Chernov 1996, 2005).

Difficulties with interpretation of ZS within the framework of DPR continue to stimulate the development of new theories. Because sometimes a large number of ZS stripes is observed, it is of interest to examine the theory proposed in LaBelle et al. (2003). This model is based on the radiation mechanism of auroral "choruses" - magnetospheric bursts observed at ground stations at frequencies of $2-4 \mathrm{MHz}$, with a fine structure similar to ZS. It is supposed that the Z-mode is radiated by the cyclotron maser mechanism (by analogy with Weengle \& Dulk 1986). Although the Z-mode at the upper hybrid frequency does not escape from the source, it can be transformed into the ordinary $(\mathrm{O})$ mode at discrete frequencies (quasi-harmonics or eigenmodes) with the presence of density enhancements with the appropriate scales.

In Sect. 2 the latest theoretical models of zebra patterns based on DPR are discussed critically. In Sect. 3, new observations of $\mathrm{ZS}$ around $3 \mathrm{GHz}$ are presented. For the analysis of two events, October 29, 2000 and April 21, 2002, all accessible ground-based (spectrometers of NAOC (China) 2,6-3,8 GHz) and satellite observational data are used (SOHO, TRACE, RHESSI). Section 4 is devoted to a discussion of these data in different models of ZS.

\section{Recent models of zebra patterns at DPR}

In two papers, Aurass et al. (2003) (I. Observations) and Zlotnik et al. (2003) (II. Source model, theory), the DPR mechanism was used for the interpretation of the short type IV burst with ZS that occurred on October 25, 1994. In the first article, the results of the force-free extrapolated magnetic field of AR 7792 in the corona are presented. The extrapolated magnetic force line starting from the head spot and used in Paper II is not connected to the radio source of ZS, since the positions of the fine structure radio sources are far to the NE of AR 7792.

The authors of Paper II do not address the conclusion of Paper I (at the end of Sect. 3.2.2) that particle acceleration took place most likely above the NE part of the loop LS1, instead of the SW part. The statement about the position of particle acceleration in the SW part of the loop LS1, made in II, contradicts Fig. 5 in (I) where it is shown that the radio sources of type III bursts at $164 \mathrm{MHz}$ are far to the NE of the bright area in which the main flare took place. The positions of radio sources of type III bursts and ZS coincided, therefore particles responsible for type III bursts should move upwards on the NE part of the loop LS1 and those responsible for ZS should move downwards in the same part of the loop.

In Paper II, the opposite problem is solved: using multiple crossings of the calculated cyclotron harmonics with the fixed frequencies of ZS stripes (at a fixed time point) a dependence $f_{\mathrm{p}}(\mathrm{h})$ is constructed (Fig. 5). The authors have considered an arbitrary selected force line in the center of the coronal loop LS1. They assert that the electron density over height follows a barometric law.

From Fig. 5 in Paper II it is visible that for the extrapolated magnetic force line the cyclotron frequency drops approximately 4 times faster than the plasma frequency (for example, for the gyroharmonic number $s=15$ ). More precisely, the scale height for the magnetic field $L_{B}=B(\mathrm{~d} B / \mathrm{d} h)^{-1}$ appears to be 4 times lower than the scale height for the electron density $L_{N}=2 N(\mathrm{~d} N / \mathrm{d} h)^{-1}$. Thus, these relations satisfy a condition for realization of DPR with a large number of harmonics $L_{N} / L_{B} \gg 1$. However, it does not satisfy a condition for the separation of zebra stripes on the continuum background, namely $\delta f / f_{B}<L_{B} /\left(L_{N}-L_{B}\right)$, at least for the nonrelativistic case.

In Appendix A, B of Paper II the main formulae for the theory of growth rates for DPR instability are presented with some newer details than Zheleznyakov \& Zlotnik (1975a) and Zheleznyakov (1995). In particular, these authors voice two objections, relating to Winglee \& Dulk (1986). First, the dispersive curves of longitudinal electrostatic waves at the frequency of DPR (shown in Fig. A1 in II) $\omega^{2}=\omega_{\mathrm{UH}}^{2}+3 k_{\perp}^{2} v_{T}^{2}$ are valid only inside the hybrid band. This cannot be extended to the band adjacent to the hybrid band from above, as done by Winglee \& Dulk (1986). Furthermore, in the footnote of Appendix A (in II) it is noted that Winglee \& Dulk (1986) considered only the first order of the small parameters $k^{2} v_{T}^{2} / \omega^{2}$ in the "thermal" dielectric tensor. That corresponds to neglecting the important resonance term. However, they do not discuss to what inaccuracies this leads in the final analysis. Thus, the main result, represented in Fig. 2 in Winglee \& Dulk (1986) is inaccurate: a paling or comb of harmonics cannot be equal in intensity for $s=6-20$.

However, in II, all formulae for similar estimations (after (B.7)) show only the qualitative behaviour of the growth rate of upper hybrid waves in DPR conditions. If we choose (in more detail) the optimal value of the wave number $k^{\text {opt }}$ (where the growth rates are maximal) for the velocity of fast particles $v_{\mathrm{e}} \approx 5 \times 10^{9} \mathrm{~cm} / \mathrm{s}$ and if we take into account the velocity dispersion $\Delta v_{\mathrm{e}} / v_{\mathrm{e}} \approx 1$ we obtain the relative bandwidth of the maximum growth rate inside the hybrid band $\delta f / f_{B}$ approximately equal to the frequency separation between stripes $\Delta f / f_{B} \approx L_{B} /\left(L_{N}-L_{B}\right) \approx 0,3$. According Stepanov et al. (1999), the frequency interval of a plasma wave excited in DPR conditions also occupies about $0,3-0,5$ of the distance $\omega_{B}$ between the adjacent harmonics. The value of $\delta f / f_{B} \approx 1 / 10$ (see Fig. B1b in II) is obtained only for an unrealistically narrow velocity dispersion $\Delta v / v \ll 1$, since in the expression (B15) the term $\Delta v / v$ is omitted (i.e. as the infinitesimal). 
Thus, for the calculated magnetic force line, the ZS stripes in Fig. 5 (in II) should merge in a continuum, and the plotting of the dependence $f(h)$ becomes almost arbitrary. The authors of II discuss merging of zebra-stripes in a continuum (in Sect. 4.2.), but do not check this condition further in the construction of Fig. 5.

Therefore the explanation of frequency drift of stripes due to a change of magnetic field and temperature cannot be accurate. The barometric formula: $f_{\mathrm{p}}=f_{\mathrm{p} 0} \exp \left(-h / 10^{4} T\right)$ (the formula (10) in II) is written by analogy to formula (3.9) in Priest (1982) for constant temperature, thus it is impossible to define from it a change of $T$. The real dependence on $T$ is given by the much more complex expression (3.8) in Priest (1982). Heating and cooling of plasma are slow processes (in the coronal loop they last some minutes), therefore it is impossible to explain a change of frequency drift of stripes on time intervals of less than $1 \mathrm{~s}$ by these processes. The authors in II have obtained an underestimated electron concentration of almost two orders relative to any other model $\left(f_{\mathrm{p} 0}=234 \mathrm{MHz}\right.$ for $\left.h=0\right)$, and the footnote on this account (that in the base of a corona other temperatures could exist) only aggravates this question, since the local parameters will not be coordinated with the global parameters for the entire corona. The authors were forced to make this assumption because of the unrealistically calculated magnetic force line.

Thus, it was necessary to determine to what other density distributions the fitting corresponds better (for example, to the Newkirk model). If it is possible to choose any force line and to vary s, this method is not suitable for the solution of such a problem.

Smooth changes of frequency drift are explained by a movement of fronts of fast particles at various angles to the magnetic force lines (Mollwo 1983, 1988). Thus, a certain restriction is imposed for the cross-section size of these fronts, so that the stripes are not merged into the continuum.

In the recent paper devoted to perfection of the DPR model of Yasnov \& Karlicky (2004), the calculations of the growth rates of the upper hybrid waves are carried out for various velocities of fast particles and various temperatures of the background plasma. From Fig. 5 in Yasnov \& Karlicky (2004), it is seen that the large width of the maximum of the growth rates does not satisfy the same condition $\delta f / f_{B}<L_{B} /\left(L_{N}-L_{B}\right)$, so that the zebra-stripes would not merge in the continuum.

In II the question of real absorption of the background continuum in dark ZS stripes is in no way discussed, although Zheleznyakov \& Zlotnik (1975a,b) relate absorptions to the negative relativistic growth rates of cyclotron waves in the hybrid band at the low frequency edge of the positive growth rates. Winglee \& Dulk (1986) do not discuss this at all due to the too large differences of the values, since the scale of negative values is about two orders less than that of the positive ones. Winglee \& Dulk (1986) conclude that in most cases the emitted harmonics overlap in the continuum radiation and zebra stripes can appear only if the maser instability is not at saturation, if the fast electrons have a loss-cone distribution and their velocities are close to $0.1 \mathrm{c}$.

Concerning the result of Zlotnik et al. (2003), namely the finding of a barometric model for the plasma frequency from

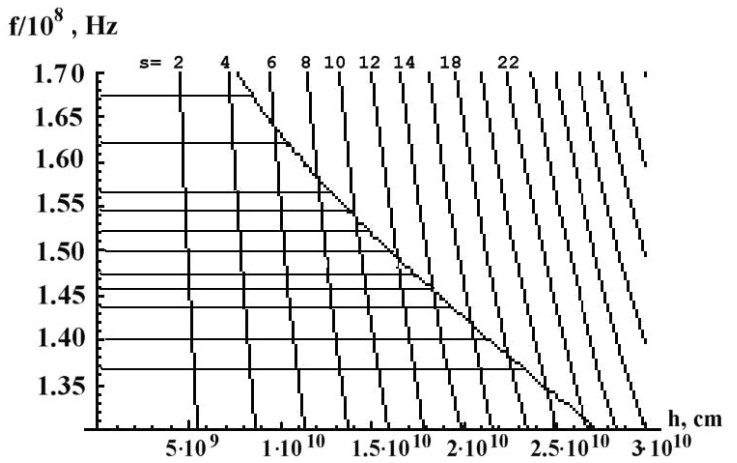

Fig. 1. Frequency $(\mathrm{Hz})$ against height in the corona $h(\mathrm{~cm})$. A grid of gyroharmonics $(s=2-22 \ldots)$ is plotted using the magnetic field model of Dulk \& McLean (1978). The inclined curve is the barometric plasma frequency dependence on height: $f_{\mathrm{p}}=f_{\mathrm{p} 0} \exp \left(-h / 10^{4} T\right)$. The horizontal lines note the frequencies of zebra stripes at a fixed time moment (10:08:23 UT), and their intersections with the barometric curve represent the DPR levels.

the observations of the ZS, it is possible to construct a similar graph, utilizing no calculated magnetic force lines (Fig. 1). It is possible to take the known model of the magnetic field in the corona, obtained from numerous radio data (McLean \& Dulk 1978),

$B=0.5 B_{0}\left(h / R_{\odot}\right)^{-1.5}, h>0.02 R_{\odot}$,

and on the analogous graph to obtain even better agreement with the barometric dependence. The horizontal lines denote the frequencies of zebra stripes at a fixed moment. On the edges of the frequency range the zebra stripes fall even closer to the levels of the DPR (harmonic from 5 through 19) than in Fig. 5a in Zlotnik et al. (2003). However, this does not mean that the barometric formula actually assigns the dependence $f_{\mathrm{p}}(h)$. For this agreement we have selected unrealistic values of $T=0.9 \times$ $10^{7} \mathrm{~K}$, although the initial frequency appears more adequate here, $f_{\mathrm{p} 0} \approx 1.05 \times 10^{9} \mathrm{~Hz}$, than in Zlotnik et al. (2003).

If the model of the field of McLean \& Dulk (1978) is accurate (it is based on a lage amount of radio data), the distribution of concentration according to the barometric formula cannot be used in the magnetic loops with plasma $\beta \ll 1$, since this formula gives the distribution of density in the gravitational field with constant temperature and without taking into account the magnetic field. The general consideration of a model with whistlers and an alternative interpretation of the zebra pattern in the event of October 25, 1994 is presented in Chernov (2005). There it is shown that the model with whistlers makes it possible to explain both the general parameters (frequency band, connection with III type bursts) and all fine effects of the behavior of the ZS on the spectrum and space drift of radio sources.

\section{Description of the selected events}

\subsection{The event of October 29, 2000}

The analysis of many cases of ZS near $3 \mathrm{GHz}$ in some events shows the full variety of fine structure similar to that seen in the metric range. In the event of October 29, 2000, for about 

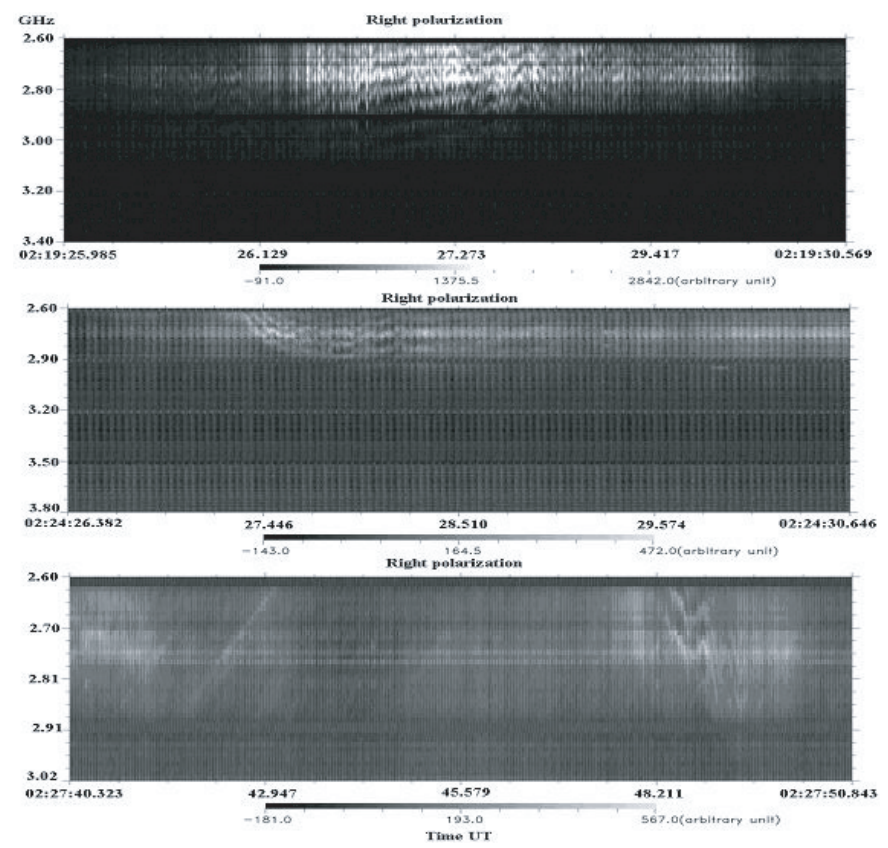

Fig. 2. Radio spectrograms of zebra patterns and fiber bursts during the evolution of the event of 29 October 2000 between 02:19-02:28 UT in right polarization recorded by the spectrometer of NAOC $(2.6-3.8 \mathrm{GHz})$. The frequency difference between the 5 regular zebra stripes in the top panel depends weakly on the frequency.

$20 \mathrm{~min}$ ZS and fiber bursts (FB) were followed by intermittent pulses of some seconds. An example of $\mathrm{ZS}$ evolution is shown in Fig. 2. This event was connected to the $\mathrm{H}_{\alpha}$ 2B M4.4 flare in AR NOAA 9209, located at S25E35. The ZS shows different frequency drifts: slow negative, positive or switches between the two. The FB often overlap the zebra pattern, and the emission frequency band is about the same for both structures. After some fluctuations of ZS a strong series of FB with different periodicity and a constant frequency drift of $-240 \mathrm{MHz} / \mathrm{s}$ was observed. The FB and ZS have about the same spectral parameters, e.g. the frequency bandwidth of emission stripes $\Delta f_{\mathrm{e}} \approx 20-30 \mathrm{MHz}$ and the frequency separation between emission lines is $50-80 \mathrm{MHz}$ in the range $2.6-2.8 \mathrm{GHz}$.

A detailed analysis of the multi-channel time profiles (see Fig. 3 in Chernov et al. 2001b) shows that the intensity level in the black stripes (between emission stripes) could be lower than the emission level of the main continuum (without ZS). Thus, the black zebra-stripes are visible not due to the absence of bright stripes, but due to an absorption of the main continuum emission (a modulation effect). In this connection the main parameter of $\mathrm{ZS}$ and $\mathrm{FB}$ is not frequency separation between emission stripes ( $\Delta f_{\mathrm{s}} \approx 60-70 \mathrm{MHz}$ on an average), but the frequency separation between the emission and the adjacent low frequency absorption with a mean value $\Delta f_{\text {ea }} \approx 30-40 \mathrm{MHz}$. Therefore we can assume that both of the fine structures have the same origin.

\subsection{The event of April 21, 2002}

The eruptive long duration X1.5 class flare event of April 21, 2002 occurred in AR 9906 close to the western solar

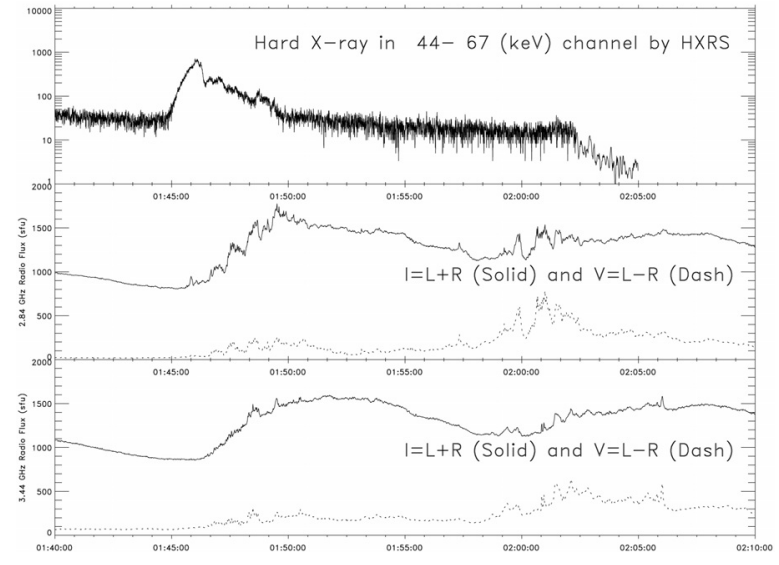

Fig. 3. Top: the hard X-ray burst in the $44-67 \mathrm{keV}$ channel of the HXRS during the period of ZS in $2.6-3.8 \mathrm{GHz}$. The Huairou radio profiles at 2.84 and $3.44 \mathrm{GHz}$ showing the radio flux intensity $(I=L+R)$ and the polarization component $(V=L-R)$ are indicated by solid and dashed lines respectively.

limb (S14W84) between 00:43 and 02:38 UT. Images of TRACE data in the EUV $195 \AA$ line show the formation of a bright post-flare arcade in the flare site (Gallagher et al. 2002). From 01:34 to at least 02:15, dark matter is seen falling onto the arcade from above. The dark matter apparently occults bright arcade loops.

The entire prolonged event was divided into two phases in the radio data and in hard X-rays: in the growing flow of soft X-rays (GOES) there was a first maximum in hard X-rays (about 01:15-01:18 UT, MTI/HXRS) and in the radio range (near 01:30 UT). In this phase the maximum energy release occurred in the decimeter wave band, which indicates magnetic reconnection at heights of the decimeter range. According to the data of radio-spectrographs (Hiraiso and IRS, Korea (Cho, personal communication)) high flux levels were recorded in the meter range (between 01:18-01:30), possibly with fine structure.

In the microwave range the maximum of the first phase was above that of the second phase. However, no fine structure in the first phase was registered around $3 \mathrm{GHz}$. The arcade of loops began to form only after approximately 01:30 UT.

The ZS are observed only during the second phase, between 01:44 and 02:05 UT, during the second phase of the HXR (MTI, HXRS) burst presented in Fig. 3. In the loop arcade we see during this phase two new bright centers as X-points of magnetic reconnection (see top left panel in Fig. 4). During the interval 01:51-01:53 UT the ZS was very weak. At this time in the TRACE $195 \AA$ image some bright centers disappeared (see right top panel in Fig. 4). The difference image between 01:50:57 and 01:48:16 shows many bright loops (middle left panel in Fig. 4), where the radio sources of ZS could be located. At the same time three new sources of HXR (RHESSI, Gallagher 2002) appeared under the mentioned two X-points of the magnetic reconnection visible in the bottom left panel of Fig. 4. Such a proposal is confirmed by radio source positions with SSRT data at $5.7 \mathrm{GHz}$ for polarized emission (the parameter $V$, see two centers shown by isolines in the bottom right panel in Fig. 4). White and black contours in the bottom left 

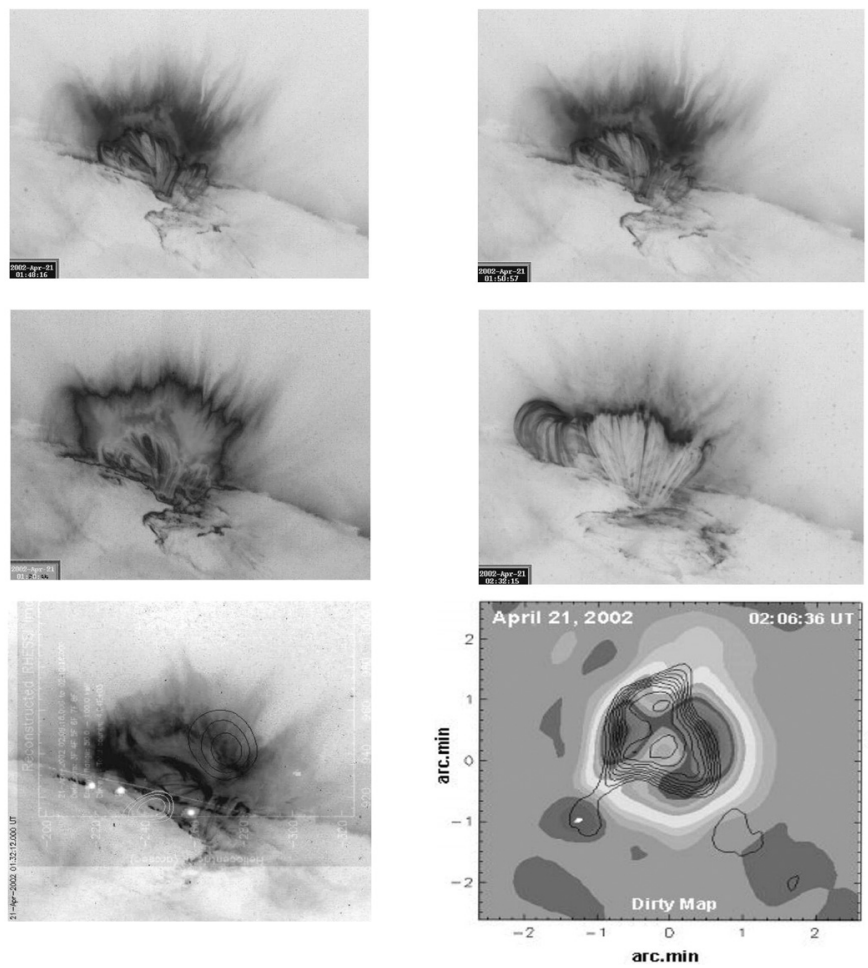

Fig. 4. The evolution of the flare of April 21, 2002 with TRACE data in EUV line $195 \AA$ and RHESSI HXR data. The difference image between 01:50:57 and 01:48:16 UT (middle left panel) shows many bright loops, where the radio sources of ZS could be located. The bottom left panel shows three new sources (white points) of HXR (RHESSI, Gallagher 2002). The bottom right panel shows radio source positions with SSRT data at $5.7 \mathrm{GHz}$ (courtesy of R. Sych) in intensity (parameter $I$, shown by the grey and black background) and in polarization (parameter $V$ shown by contours).

panel of Fig. 4 show source positions of RHESSI/HXR energy channels respectively at 50-100 and 12-25 Kev (Gallagher 2002). Radio sources of continuum emission in intensity with SSRT data (parameter $I$ ) have approximately the same positions (the lower and upper sources shown by the grey and black background in the bottom right panel of Fig. 4).

After 02:09 UT the arcade of bright loops in the line $195 \AA$ rose to high altitudes (middle right panel in Fig. 4), and the maximum of activity was displaced into the decimeter range.

All series of the ZS in this event represented zebra stripes with negative frequency drift, which is more inherent to fibers. In Figs. 5 and 6 two intervals with the most pronounced ZS are shown, taken with the NAOC spectrometer at 2.6-3.8 GHz. In the first fragment of $20 \mathrm{~s}$ duration from 01:48:18 UT 34 zebra stripes are counted in the range $2.6-3.8 \mathrm{GHz}$ with a barely noticeable increase in frequency separation between stripes from $27 \mathrm{MHz}$ at $2.8 \mathrm{GHz}$ to $43 \mathrm{MHz}$ at $3.7 \mathrm{GHz}$. It is remarkable that the relative value of the frequency separation proves to be about the same as in the meter range $\Delta f / f \approx 0.012$.

The frequency drift of zebra stripes was negative and almost constant $\approx-120 \mathrm{MHz} / \mathrm{s}$ between $3.8-3.4 \mathrm{GHz}$, which is more characteristic of fibers. At the low frequency part of the spectrum a small deceleration of the frequency drift is observed. However, each 1-2 s, stripes undergo failures and
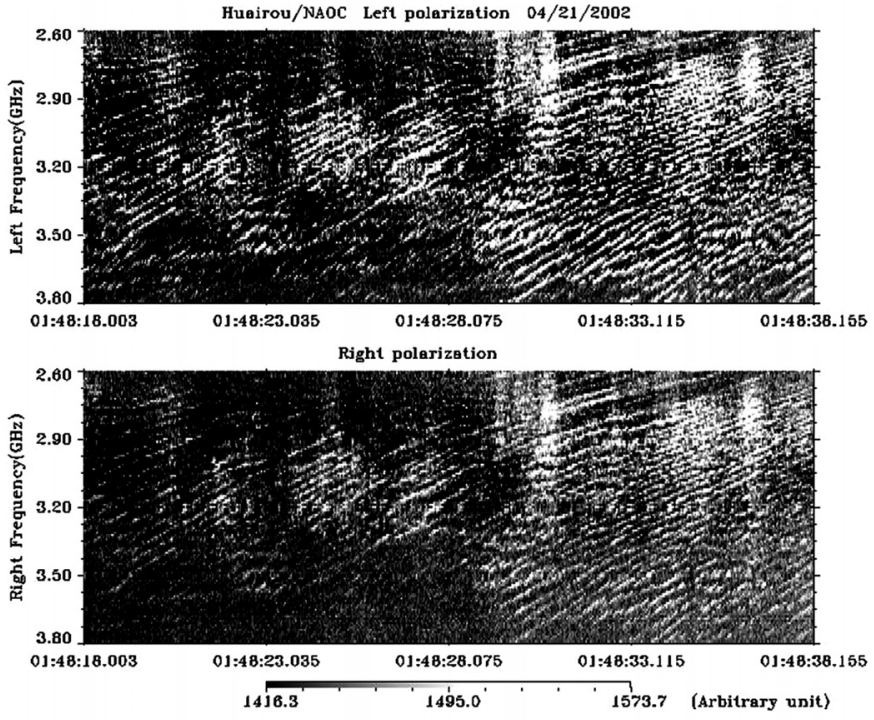

Huairou/NAOC Left polarization

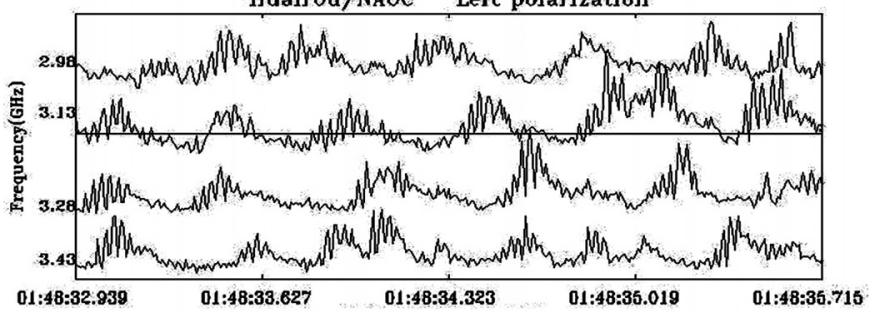

Fig. 5. Zebra structure in the event of April 21, 2002. The two upper panels show the Huairou radio spectrograms in the $2.6-3.8 \mathrm{GHz}$ range in the left and right polarization components with a zebra pattern at 01:48:18-01:48:38 UT, in which 34 stripes can be distinguished and whose frequency separation grows smoothly with frequency. The lower panel shows profiles of the intensities at four fixed frequencies. The profiles confirm the spike-like structure of each zebra stripe in emission. The dark stripes do not contain a spike-like structure, and the radiation level proves to be lower than the average level of continuum, shown by the straight line at a frequency of $3.13 \mathrm{GHz}$.

jumps of frequency drift, covering several adjacent stripes with a small time delay. Sometimes shifts in the frequency lead to merging or splitting of stripes. Such effects are well known in the meter wave band. In the bottom of Fig. 5 a short fragment of intensity time profiles of about $3 \mathrm{~s}$ duration is shown at four frequencies. A detailed analysis of multichannel time profiles shows that each zebra stripe in emission has a spike-like structure. In the dark stripes (between the bright zebra stripes) the emission level can be lower than the level of burst background (lower than the average level of continuum, shown by a straight line at $3.12 \mathrm{GHz}$ ). Thus the presence of dark stripes is connected not with the absence of bright stripes (in emission), but with an absorption of background microwave emission. The polarization degree was weakly left-handed. We propose that two polarized radio sources at $5.7 \mathrm{GHz}$ were located above the leading spot (the left polarized source) and above the tail spot (the right source in the bottom right panel of Fig. 4). Therefore due to the limb position of the AR it is difficult to precisely define the magnetic polarity of the corresponding radio sources to define the type of radio wave. 

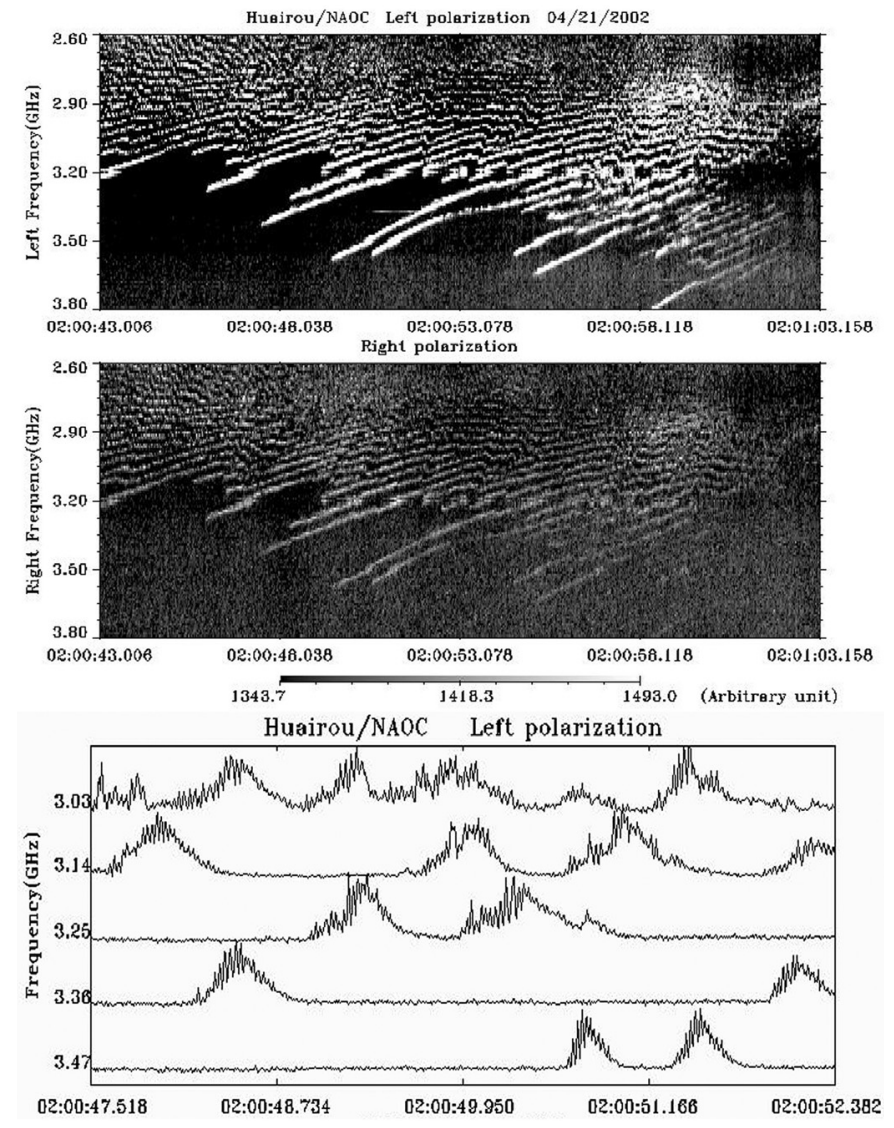

Fig. 6. Zebra structure in the event of April 21, 2002 at 02:00:43-02:01:03 UT. The zebra stripes on the high-frequency edge of the spectrum are more similar to isolated fibers without any strict periodicity. The multichannel time profiles (in the bottom panel) show stripes in emission consisting of spikes as before. However, in the dark stripes the residual emission remains at a fixed level.

The fragment in Fig. 6 shows some changes of ZS properties: the polarization degree becomes stronger and it grows with time and frequency; the frequency drift almost stops in the lowfrequency part of the spectrum (sometimes the stripes are almost parallel to the time axis); however, in the high-frequency part of the band they are more similar to isolated fibers with a negative frequency drift (there is no strict periodicity). The multichannel time profiles (in the bottom panel of Fig. 6) show that stripes in emission consist as before of spikes, however, in the dark stripes, deep dips in emission are not observed; the residual emission remains at a fixed level.

$\mathrm{ZS}$ was also observed in the pulsating regime, but almost all series of ZS were prolonged; in the examples in Figs. 5 and 6 the duration exceeded $1 \mathrm{~min}$. The evolution of zebra stripes in several series began from a cloud of millisecond spikes with the gradual shaping of zebra stripes, as is shown in Fig. 7.

\section{Discussion and conclusions}

\subsection{The event of October 29, 2000}

The relative frequency parameters of ZS in microwave and meter wave bands appear identical. This testifies to the similar nature of the formation of fine structure in different frequency
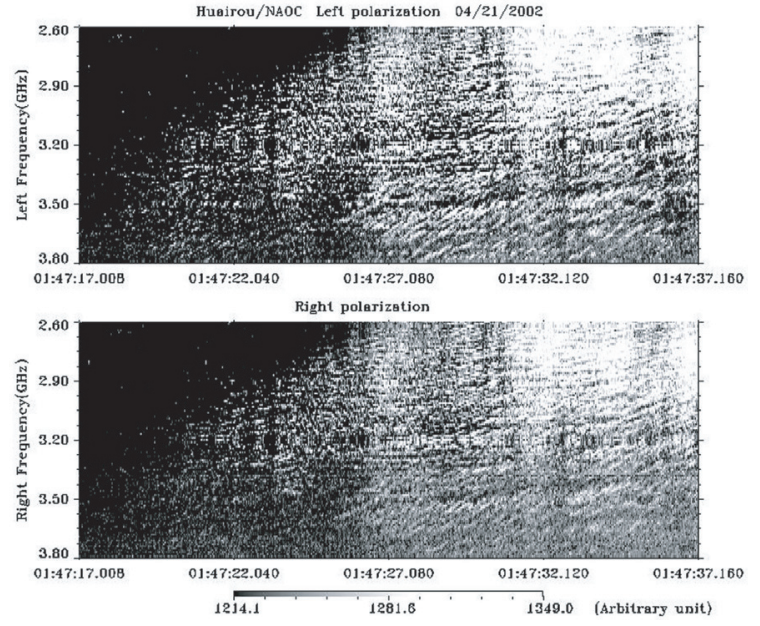

Fig. 7. Some series of ZS began from a cloud of millisecond spikes with gradual formation of zebra stripes.

ranges. Since ZS and FB display similar spectral characteristics, we will consider both structures to be manifestations of whistlers in the radio source, due to their interactions with electrostatic plasma waves: $l+w \rightarrow t$, with both waves being excited by the same fast particles in hot flare loops, which have an anisotropic loss-cone distribution. The computations of Yasnov et al. (2000) show a growth in the whistler increment with temperature of the background plasma in the flare region, with the growth in cyclotron decay being compensated by the decrease in the whistler frequency with a maximum increment at $0.1 \omega_{\mathrm{Be}}$. The mean duration of a series of zebra structures and fiber bursts was about two seconds, and the propagation of whistlers without damping over this time places a constraint on the whistler increment of $<0.5 \mathrm{~s}^{-1}$. This constraint can be used to estimate the magnetic field strength in the region in which the flare is generated. Electron temperatures in the range 2-20 MK yield magnetic fields of $B=125-190 \mathrm{G}$ for regions in which the electron density is $(8-18) \times 10^{10} \mathrm{~cm}^{-3}$.

These rough estimates coincide with estimates derived from the frequency drift rate of the fiber bursts observed at the same time at these same frequencies, based on the formula $B=15.43(\ln f-3)^{-2} \mathrm{~d} f / \mathrm{d} t($ Elgarøy 1982), which was obtained for a 60 -fold Newkirk model and a whistler frequency of $\omega_{w}=0.1 \omega_{B}$. In the model with the whistlers we can also verify the obtained values of B using the frequency separation between the maximum in the emission and the adjacent minimum in the absorption $\left(\Delta f_{\mathrm{ea}}\right)$. This value must be equal to the frequency of whistlers, which is selected to be equal to 0.1 of the cyclotron frequency (at which the increment of whistlers is maximum). Thus, $B=\Delta f_{\text {ea }} /(0.1 \times 2.8)$ and for the observed value $\Delta f_{\text {ea }} \approx 40 \mathrm{MHz}$ we can estimate $B \approx 140 \mathrm{G}$. This value is close to the values of the field, obtained above by two other methods, which confirms the reliability of the determination of the magnetic field in the model with whistlers.

If we estimate the magnitude of $B$ in the new model of LaBelle et al. (2003), assuming that $\Delta f_{\mathrm{s}} \approx 0.02 f_{\mathrm{Be}}$, we obtain for $\Delta f_{\mathrm{s}}=80 \mathrm{MHz}$ implausibly high values of $B \sim 1500 \mathrm{G}$, close to photosphere values. 


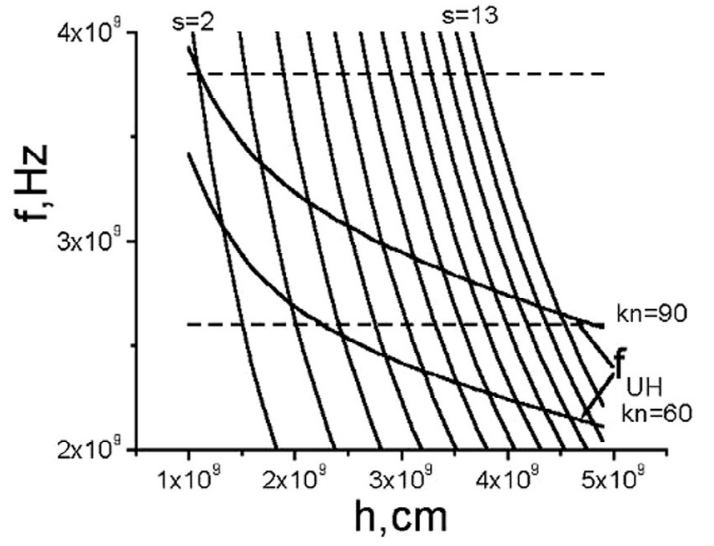

Fig. 8. Dependences of upper hybrid and cyclotron frequencies on the height in the corona in the magnetic field model of Dulk \& McLean (1978), which is derived from radio data $\left(B(G)=0.5\left(R / R_{\odot}-1\right)^{-1.5}\right.$, $\left.1.02=R / R_{\odot}=10\right)$, and the electron density model of Newkirk multiplied by $k n$ : $\left(n=k n \cdot 8.26 \times 10^{4} \times 10^{4.32 / h} \mathrm{~cm}^{-3}\right)$. The horizontal dashed lines delineate the range of the NAOC radio spectrograph, 2.6-3.8 GHz.

The alternative model based on the generation of plasma waves at the upper-hybrid frequency $\omega_{\mathrm{UH}}$ under the conditions appropriate for DPR was also considered. In the DPR- model all estimations of the field depend strongly (even for the order of magnitude) on the unknown relationship between the height scales in the corona of the density and magnetic field. We can see from Fig. 8 that the main inadequacy of this model is associated with the increase in the frequency separation of the emission stripes $\Delta f_{\mathrm{s}}$ with increasing frequency: the model predicts an increase from $60 \mathrm{MHz}$ at $2.7 \mathrm{GHz}$ to $\sim 450 \mathrm{MHz}$ at $3.8 \mathrm{GHz}$, which is not generally observed. Usually, the frequency separation only slightly increases with frequency.

The conditions of DPR are probably realized rarely in the flare region with a set of thin magnetic loops, in which the magnetic field and density change insignificantly with the height along the loops (Aschwanden et al. 1999; Aschwanden 2002). However, if they fall in a stable magnetic trap, one may expect the stable emission of stripes, most likely during the entire event. Such stable stripes in emission were observed, for example, in the decimeter range in the event of February 17, 1992 (Chernov et al. 1998, evolving emission lines, EEL). The EEL with slow wave frequency drift of one or three harmonics during more than $2 \mathrm{~h}$ satisfies the mechanism for DPR.

\subsection{The event of April 21, 2002}

Figures 3 and 4 show the main flare moments at which a zebra pattern appears. First, the interval of a zebra pattern synchronizes precisely with an HXR burst (44-67 keV, HXRS). Second, the two most powerful series of ZS represented in Figs. 5 and 6 coincide in time with the appearance of two new HXR sources (RHESSI data in the lower left panel of Fig. 4), whose positions coincide with two maximum radio sources (SSRT data) in the polarized radiation. Thus, the formation of zebra stripes is related to the presence of fast particles in the loop arcade, including two bright nodes in the formation
$X$-points of magnetic reconnection, seen in the difference image in Fig. 4. The emission in the FeXII line at $195 \AA$ shows only bright loops with a temperature of $1.8 \times 10^{6} \mathrm{~K}$, but radio sources at $5.7 \mathrm{GHz}$ (SSRT) and $17 \mathrm{GHz}$ (Nobeyama) in the polarized radiation were located near two bright nodes, in which the bright intersecting loops had a configuration of the form of singular $X$-points.

The dark material on top is probably overheated flare plasma (to $50 \mathrm{MK}$ ) ejected from the region of maximum energy release at heights in the decimeter range. This prolonged flow of overheated plasma (from 01:34 through 02:15 UT)likely served as the source of heating of the entire arcade (not only the mentioned nodes with the $X$-points), since the duration of its precipitation tightly covered the interval of fine structure excitation.

It would be natural to connect radio sources with these bright loops. However, the large number of loops with bright apexes in a narrow height interval casts doubt on the possibility of realization of DPR-conditions in a wide interval of heights. In this connection, the example of the zebra structure shown in Fig. 5 is noteworthy since it shows that the DPR-model is not able to explain the presence of simultaneous emission in 34 zebra stripes with virtually identical intensities in the range 2.6-3.8 GHz.

At the same time, this situation is realistic in a whistler model, where strict periodicity of the stripes is specified by the whistler excitation mechanism itself (fluctuations in the instability under the action of quasi-linear effects, independent of the models for density and magnetic field in the corona) (Chernov 2005). Frequently the zebra stripes are more similar to isolated fibers, as, for example, on the high-frequency edge of the spectrum in Fig. 6. Therefore we could consider both fine structures in a unique model as manifestations of the whistler mechanism. The isolated packages of whistlers are excited more effectively below, near magnetic mirrors, and they propagate upward along the magnetic loops. Approaching the singular points ( $X$-points), whistlers gradually fill the entire radio source with almost standing periodic wave packets, that also explain the presence of stripes barely drifting in frequency at the low-frequency edge of the spectrum in Fig. 6.

The new model of LaBelle et al. (2003) can theoretically explain all the parameters of zebra structures and as well as the large number of zebra pattern stripes originating from a point radio source. However, the stable appearance of series of zebra structures in a pulsating regime cannot be understood since this model predicts a strong dependence of the frequency separation and the number of zebra stripes on the parameters of the inhomogeneities, while we observe only a very small growth in the frequency separation with increasing frequency. The parameters of inhomogeneities such as propagating ionsound waves cannot remain so similar over a wide range of frequencies (interval of heights) and over extended periods of time. Furthermore, we need to explain the large intensity of all zebra stripes from numerous point radio sources, exciting the total (incoherent) emission i.e., the spectrum of the stripes would be expected to blend into the continuum.

The frequency profiles in Figs. 5 and 6 demonstrate rapid pulsations in the bright zebra-pattern stripes with a 
well-defined period of $30 \mathrm{~ms}$. Thus, these new observations support the conclusions of Chernov et al. (2003) that the ZS at centimeter wavelengths always have a fine spike-like structure, which can be explained by whistler models for zebra structure.

Figure 7 shows that zebra stripes are gradually formed in a cloud of spikes. Therefore it is possible to assume that the emission of spikes is a primary process. In the dm- range the analogous conversion was observed by Kuijpers et al. (1981). They assumed that spikes and zebra patterns can be excited by the same mechanism. On the basis of our previous results (Chernov et al. 2003), we can assume that near the flare region spikes can be excited by the nonlinear mechanism of interaction of plasma Langmuir waves with ion-sound waves. The ionic sound can be always present in shock wave fronts, departing from regions of magnetic reconnection (Chernov et al. 2001a). However, zebra stripes appear with the excitation of whistlers by fast particles with a loss-cone distribution, forming only after the reflection of particles from the magnetic mirrors. Thus, a certain delay is permissible.

If we determine the magnetic field strength of the frequency separation between the maxima in emission and absorption in one stripe $\left(\Delta f_{\mathrm{ea}}\right)$, for the moment 01:48:34 UT, we obtain a small value of $B \approx 90 \mathrm{G}$.

After 02:00 UT we do not have the possibility to estimate $B$, since the zebra stripes did not show noticeable absorption, even in the single stripes at the HF edge. A decrease in the intensity of plasma waves due to scattering of fast particles on the whistlers in the periodic wave packets is the basic reason for absorptions. Therefore assume that in this case the particles and waves diverged in space, which is allowed with rapid particle injection and the emission of whistlers with the normal Doppler effect (in a regime of weak diffusion). It is not excluded that the large number of stripes with a narrow frequency separation is the result of the imposition of emission from the different loops due to the observation of the projection of radio sources at the solar limb. In this case the polarization will most likely be determined by the propagation conditions of radio emission perpendicular to the magnetic field.

Thus, new data on zebra structure and fiber bursts at centimeter wavelengths show that they have similar structures to those observed at meter wavelengths. ZS was not observed at frequencies higher than 5.5-6 GHz. The radiation of electrostatic plasma waves at higher frequencies is probably suppressed in the dense flare plasma. A unified model for zebra structure and fiber bursts involving whistlers can yield realistic values for the magnetic field strength, $B \sim 140-160 \mathrm{G}$, at a plasma level of about $3 \mathrm{GHz}$. Using realistic dependences for the electron density and magnetic field, the DPR-model for zebra structure predicts a frequency dependence of the frequency separation between stripes that is much stronger than is observed.

Acknowledgements. This research was supported by the Russian Foundation of Basic Research, grants No. 02-02-16201, 04-02-39015.
G.P.Ch. thanks Dr. L.V. Yasnov for the help in some calculations. G.P.Ch. is grateful also for the support of the Chinese Academy of Sciences and NSF of China that enabled him to work with colleagues at NAOC. The Chinese authors acknowledge support of NSFC and Ministry of Sciences and Technology of China (G2000078403). The TRACE and RHESSI data were obtained from the TRACE and RHESSI databases.

\section{References}

Aschwanden, M. J. 2002, Particle acceleration and kinematics in solar flares (Dordrecht: Kluwer Ac. Publ.)

Aschwanden, M. J., Newmark, J. S., Delabouniere, J.-P., et al. 1999, ApJ, 515, 842

Aurass, H., Klein, K.-L., Zlotnik, E. Ya., \& Zaitsev, V. V. 2003, A\&A, 410, 1001

Chernov, G. P. 1996, Astron. Rep., 40, 561

Chernov, G. P. 2005, Plasma Phys. Rep., 31, 314

Chernov, G. P., Markeev, A. K., Poquerusse, M., et al. 1998, A\&A, 334,314

Chernov, G. P., Fu, Q. J., Lao, D. B., \& Hanaoka, Y. 2001a, Sol. Phys., 201, 153

Chernov, G. P., Yasnov, L. V., Yan, Y. H., \& Fu, Q. J. 2001b, Chin. J. Astron. Astrophys., 1, 525

Chernov, G. P., Yan, Y., \& Fu, Q. 2003, A\&A, 406, 1071

Dulk, G. A., \& McLean, D. J. 1978, Sol. Phys., 57, 279

Elgarøy, Ø. 1959, Nature, 184, 887

Elgarøy, Ø. 1982, Intermediate drift bursts, Rep., 53, ITA, Univ. Oslo

Fu, Q. J., Qin, J. H., Ji, H. R., \& Pei, L. B. 1995, Sol. Phys., 160, 97

Gallagher, P. T., Dennis, B. R., Krucker, S., Schwartz, R. A., \& Tolbert, A. K. 2002, Sol. Phys., 210, 341

Karlický, M., Bárta, M., Jiřička, K., et al. 2001, A\&A, 375, 638

Kuijpers, J. 1975, Collective wave-particle interactions in solar type IV radio sources, Utrecht University

Kuijpers, J. 1980, Theory of type IV dm bursts, in Radio Physics of the Sun, ed. M. R. Kundu, \& T. E. Gergely, 341

Kuijpers, J., Van der Post, P., \& Slottje, C. 1981, A\&A, 102, 331

Ledenev, V. G., Karlický, M., Yan, Y., \& Fu, Q. 2001, Sol. Phys., 202, 71

LaBelle, J., Treumann, R. A., Yoon, P. H., \& Karlicky, M. 2003, ApJ, 593, 1195

Mollwo, L. 1983, Sol. Phys., 83, 305

Mollwo, L. 1988, Sol. Phys., 116, 323

Sawant, H. S., Karlicky, M., Fernandes, F. C. R., \& Cecattto, J. R. 2002, A\&A, 396, 1015

Slottje, C. 1972, Sol. Phys., 25, 210

Slottje, C. 1981, Atlas of fine structures of dynamic spectra of solar type IV-dm and some type II bursts, Utrecht Observatory

Winglee, R. M., \& Dulk, G. A. 1986, ApJ, 307, 808

Yasnov, L. V., Chernov, G. P., Yan, Y., \& Fu, Q. 2002, Proc. 10th, European SPM, Solar Variability: From Core to Outer Frontiers, Prague, ESA SP-506, 791

Zheleznyakov, V. V. 1995, Radiation in Astrophysical Plasmas (Dordrecht: Kluwer Academic Publisher)

Zheleznyakov, V. V., \& Zlotnik, E. Ya. 1975a, Sol. Phys., 43, 431

Zheleznyakov, V. V., \& Zlotnik, E. Ya. 1975b, Sol. Phys., 44, 461

Zlotnik, E. Ya., Zaitsev, V. V., Aurass, H., \& Hofmann, A. 2003, A\&A, 410,1011 